\title{
Linear Stability Analysis of Double Diffusive Convection in a Horizontal Sparsely Packed Rotating Anisotropic Porous Layer in Presence of Soret Effect
}

\author{
S. N. Gaikwad ${ }^{+1}$ and S. S. Kamble ${ }^{2}$ \\ ${ }^{1}$ Department of Mathematics, Gulbarga University, Jnana Ganga Campus, Gulbarga 585 106, India. \\ ${ }^{2}$ Department of Mathematics, Government First Grade College, Chittapur - 585 211, India. \\ †Corresponding Author Email: sngaikwad2009@yahoo.co.in
}

(Received March 31, 2013; accepted June 9, 2013)

\begin{abstract}
In this paper, we have investigated theoretically the effect of Soret parameter on the onset of double diffusive rotating anisotropic convection in a horizontal sparsely packed porous layer using linear stability theory which is based on the usual normal mode technique. The Brinkman model that includes the Coriolis term is employed for the momentum equation. The effect of anisotropy parameters, Soret parameter, solute Rayleigh number, Taylor number, Lewis number, Darcy and Darcy Prandtl number on stationary and oscillatory convection is shown graphically.
\end{abstract}

Keywords: Soret parameter, Rotation, Anisotropy, Double diffusive convection.

\section{NOMENCLATURE}

$a \quad$ wave number

$c \quad$ specific heat of solid

$c_{p} \quad$ specific heat of fluid at constant pressure

$d$ height of the porous layer

$D_{1}$ anisotropic thermal diffusion tensor,

$D_{1 x} \mathbf{i i}+D_{1 y} \mathbf{j} \mathbf{j}+D_{1 z} \mathbf{k k}$

$D_{11}$ soret coefficient

$D_{2}$ solute Diffusivity

$D a$ Modified Darcy number, $\mu_{e} K_{Z} / \mu_{f} d^{2}$

g gravitational acceleration, $(0,0,-g)$

$K$ inverse anisotropic permeability tensor, $K_{x}^{-1} \mathbf{i}+K_{y}^{-1} \mathbf{j} \mathbf{j}+K_{z}^{-1} \mathbf{k k}$

$l, m$ horizontal wavenumbers

Le Lewis number, $D_{1 z} / D_{2}$

$p \quad$ pressure

$P r_{D} \quad$ Darcy-Prandtl number, $\gamma \varepsilon v d^{2} / K_{z} D_{1 z}$

$\boldsymbol{q}$ velocity vector, $(u, v, w)$

$R_{T}$ thermal Rayleigh number, $\beta_{T} g \Delta T d K_{z} / v D_{1 z}$

$R_{S} \quad$ solute Rayleigh number, $\beta_{S} g \Delta S d K_{z} / v D_{1 z}$ $\varepsilon \quad$ porosity

$\rho_{0}$ reference density

$\eta \quad$ thermal anisotropy parameter, $D_{1 x} / D_{1 z}$

$\mu_{f} \quad$ fluid viscosity

$\mu_{e} \quad$ effective viscosity

$V \quad$ kinematic viscosity

$\Theta \quad$ dimensionless amplitude of temperature perturbation

$\sigma$ growth rate

$\Omega$ angular velocity of rotation, $(0,0, \Omega)$

$\xi \quad$ mechanical anisotropy parameter, $K_{x} / K_{z}$

$\lambda$ normalized porosity, $\varepsilon / \gamma$

$\Phi$ dimensionless amplitude of concentration perturbation

$\psi \quad$ stream function

Other symbols

$$
\begin{array}{ll}
\nabla_{h}^{2} & \frac{\partial^{2}}{\partial x^{2}}+\frac{\partial^{2}}{\partial y^{2}} \\
\nabla^{2} & \nabla_{h}^{2}+\frac{\partial^{2}}{\partial z^{2}}
\end{array}
$$

Subscripts

$b \quad$ basic state 
S. N. Gaikwad and S. S. Kamble / JAFM, Vol. 7, No. 3, pp. 459-471, 2014.

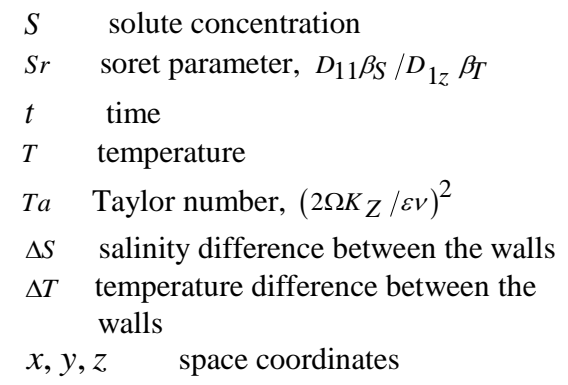

\section{Greek symbols}

$$
\begin{array}{ll}
\beta_{T} & \text { thermal expansion coefficient } \\
\beta_{S} & \text { solute expansion coefficient } \\
\gamma & \text { ratio of specific heat, } \frac{(\rho c)_{m}}{\left(\rho c_{p}\right)_{f}}
\end{array}
$$

\section{INTRODUCTION}

A double diffusive process is one that is driven by the presence of two different substances that diffuse at different rates. Double diffusion was first explored because of its application to oceanic phenomena. In the ocean, the density of water is governed primarily by temperature and salinity. The applications of the present study has recently increased in view of its potential occurrence in nature and wide range of applications such as highquality crystal production, liquid gas storage, migration of moisture in fibrous insulation, transport of contaminants in saturated soil, solidification of molten alloys, and geothermally heated lakes and magmas, underground disposal of nuclear wastes, liquid re-injection, electro-chemical and drying processes.

A comprehensive review of the literature concerning double diffusive natural convection in a fluid-saturated porous medium may be found in the book by Nield and Bejan (2006). Useful review articles on double diffusive convection in porous media include those by Trevisan and Bejan (1999), Mojtabi and Charrier-Mojtabi (2005) and Mamou (2002), Vafai(2005) and Vadasz(2008), Ingham and Pop(2005).

The onset of double diffusive convection in a horizontal porous layer has been investigated by Rudraiah et al. (1982) using a weak non-linear theory. Finite amplitude double diffusive convection near the threshold of both stationary and oscillatory instabilities in a binary mixture was investigated by Brand and Steinberg (1983).The linear stability analysis of the thermosolutal convection in a sparsely packed porous layer was made by Poulikakos (1986) using the DarcyBrinkman model. Small amplitude nonlinear solutions in the form of standing and traveling waves and the transition to finite amplitude convection, as predicted by bifurcation theory, were studied by Knobloch (1986). The double diffusive convection in porous media in the presence of Soret

$\begin{array}{ll}c & \text { critical } \\ f & \text { fluid } \\ h & \text { horizontal } \\ s & \text { solid } \\ m & \text { porous medium } \\ 0 & \text { reference value }\end{array}$

\section{Superscripts}

$\begin{array}{ll}* & \text { dimensionless quantity } \\ { }^{*} & \text { perturbed quantity } \\ \text { osc } & \text { oscillatory state } \\ \text { st } & \text { stationary state }\end{array}$

and Dufour coefficients has been analyzed by Rudraiah and Malashetty (1986).

Murray and Chen (1989) have extended the linear stability theory, taking into account effects of temperature-dependent viscosity and volumetric expansion coefficients and nonlinear basic salinity profile. Double diffusive fingering convection in a porous medium with horizontally periodic boundary conditions was studied by Chen and Chen (1993). Malashetty (1993) made a linear stability analysis to determine the effects of anisotropic thermo convective currents on the double diffusive convection in a sparsely packed porous medium.

Straughan and Hutter (1999) have investigated the double diffusive convection with Soret effect in a porous layer using Darcy-Brinkman model and derived a priori bounds. An analytical and numerical study of double diffusive convection with parallel flow in a horizontal sparsely packed porous layer under the influence of constant heat and mass flux was performed using a Brinkman model by Amahmid et al. (1999).

Bahloul et al. (2003) have carried out an analytical and numerical study of the double diffusive convection in a shallow horizontal porous layer under the influence of Soret effect. Hill (2005) performed linear and nonlinear stability analyses of double diffusive convection in a fluid saturated porous layer with a concentration based internal heat source using Darcy's law. Double diffusive natural convection within a multilayer anisotropic porous medium is studied numerically and analytically by Bennacer et al. (2005). Mansour et al. (2006) have investigated the multiplicity of solutions induced by thermosolutal convection in a square porous cavity heated from below and subjected to horizontal concentration gradient in the presence of Soret effect.

There are only few studies available on double diffusive convection in the presence of rotation. Chakrabarti and Gupta (1981) have analyzed the nonlinear thermohaline convection in a rotating porous medium. The effect of rotation on linear and 
non-linear double diffusive convection in a sparsely packed porous medium was studied by Rudraiah et al. (1986).

Anisotropy is generally a consequence of preferential orientation or asymmetric geometry of porous matrix or fibers and is in fact encountered in numerous systems in industry and nature. Anisotropy is particularly important in a geological context, since sedimentary rocks generally have a layered structure; the permeability in the vertical direction is often much less than in the horizontal direction. The review of research on convective flow through anisotropic porous media has been well documented by McKibbin (1992) and Storesletten (2004). Castinel and Combarnous (1974) have conducted an experimental and theoretical investigation on the Rayleigh-Benard convection in an anisotropic porous medium. Epherre (1977) extended the stability analysis to a porous medium with anisotropy in thermal diffusivity also.

Recently many authors have studied the effect of anisotropy and/or rotation on the onset of convection in a horizontal porous layer (see e.g. Govender; 2007, Malashetty and Swamy; 2007). There appears to be only couple of studies available on the double diffusive convection in an anisotropic porous medium with rotation ( Patil et al., 1989) and without rotation (Tyvand; 1980).

Recently Gaikwad and Kamble (2012) have investigated the Soret effect on double diffusive convection in a horizontal sparsely packed porous layer. Rani and Reddy (2013) have studied the Soret and Dufour Effects on Transient Double Diffusive Free Convection of Couple-stress Fluid past a Vertical Cylinder. The effect of rotation on the onset of double diffusive convection in a sparsely packed anisotropic porous layer is studied by Malashetty and Begum (2011). A very little attention has been devoted to the study of double diffusive convection in rotating porous medium including the mechanical and thermal anisotropic effects in presence of Soret effect. Therefore, the objective of the present study is to investigate the combined effect of rotation and anisotropy in presence of Soret effect on the double diffusive convection in a horizontal sparsely packed porous layer using linear stability analyses.

\section{GOVERNING EQUATIONS}

Consider a horizontal sparsely packed anisotropic porous layer, saturated with Boussinesq fluid of infinite horizontal extent confined between parallel, stress-free planes $z=0$ and $z=d$ subject to rotation and maintained at constant temperatures $T_{0}+\Delta T$ and $T_{0}$ with solute concentration $S_{0}+\Delta S$ and $S_{0}$ respectively. A Cartesian frame of reference is chosen with $x$-and $y$-axes at the lower boundary plane and $z$-axis directed vertically upwards in the gravity field. The axis of rotation is assumed to coincide with the $z$-axis with a constant angular velocity $\boldsymbol{\Omega}=(0,0, \Omega)$. The
Darcy-Brinkman model that includes the Coriolis term is used for the momentum equation. The velocities are assumed to be small so that the advective and Forchheimer inertia effects are ignored. The Boussinesq approximation, which states that the variation in density is negligible every where in the conservations except in the buoyancy term, is assumed to hold. With these assumptions the basic governing equations are

$\nabla . \boldsymbol{q}=0$

$$
\begin{aligned}
& \rho_{0}\left(\frac{1}{\varepsilon} \frac{\partial \boldsymbol{q}}{\partial t}+\frac{2}{\varepsilon} \mathbf{\Omega} \times \boldsymbol{q}\right)+\mu_{f} \mathbf{K} \cdot \boldsymbol{q} \\
& =-\nabla p+\rho \mathbf{g}+\mu_{e} \nabla^{2} \boldsymbol{q} \\
& \gamma \frac{\partial T}{\partial t}+(\boldsymbol{q} \cdot \nabla) T=\nabla \cdot\left(D_{1} \cdot \nabla T\right) \\
& \varepsilon \frac{\partial S}{\partial t}+(\boldsymbol{q} \cdot \nabla) S=D_{2} \nabla^{2} S+D_{11} \nabla^{2} T \\
& \rho=\rho_{0}\left(1-\beta_{T}\left(T-T_{0}\right)+\beta_{S}\left(S-S_{0}\right)\right)
\end{aligned}
$$

The basic state of the fluid is assumed to be quiescent. The quantities of the basic state are given by

$$
\begin{aligned}
& \mathbf{q}_{b}=(0,0,0), p=p_{b}(z), T=T_{b}(z), \\
& S=S_{b}(z), \rho=\rho_{b}(z)
\end{aligned}
$$

which satisfy the equations

$$
\begin{aligned}
& \frac{d p_{b}}{d z}=-\rho_{b} g, \frac{d^{2} T_{b}}{d z^{2}}=0, \frac{d^{2} S_{b}}{d z^{2}}=0, \\
& \rho_{b}=\rho_{0}\left(1-\beta_{T}\left(T_{b}-T_{0}\right)+\beta_{S}\left(S_{b}-S_{0}\right)\right)
\end{aligned}
$$

On the basic state we superpose small perturbations around the basic solutions in the form

$$
\begin{aligned}
& \mathbf{q}=\mathbf{q}_{b}+\mathbf{q}^{\prime}, T=T_{b}+T^{\prime}, S=S_{b}+S^{\prime}, \\
& p=p_{b}+p^{\prime}, \rho=\rho_{b}+\rho^{\prime}
\end{aligned}
$$

where the primes indicate perturbations.

Substituting Eq. (8) into Eqs. (1) to (5) and using the basic state Eqs. (7) and transformations

$$
\begin{aligned}
& \left(x^{\prime}, y^{\prime}, z^{\prime}\right)=\left(x^{*}, y^{*}, z^{*}\right) d, t^{\prime}=t^{*}\left(\gamma d^{2} / D_{1 z}\right), \\
& \left(u^{\prime}, v^{\prime}, w^{\prime}\right)=\left(D_{1 z} / d\right)\left(u^{*}, v^{*}, w^{*}\right), \\
& T^{\prime}=(\Delta T) T^{*}, S^{\prime}=(\Delta S) S^{*}
\end{aligned}
$$

to render the equations dimensionless we obtain(after dropping the asterisks for simplicity

$$
\begin{aligned}
& w\left[\frac{1}{P r_{D}} \frac{\partial}{\partial t} \nabla^{2}+\frac{1}{\xi} \frac{\partial^{2}}{\partial z}-D a \nabla^{4}+\nabla_{h}^{2}\right] \times \\
& {\left[T a \frac{\partial^{2}}{\partial z^{2}}+\right.} \\
& {\left[\left(\frac{1}{P r_{D}} \frac{\partial}{\partial t}+\frac{1}{\xi}-D a \nabla^{2}\right)\right]} \\
& =\left(\frac{1}{P r_{D}} \frac{\partial}{\partial t}+\frac{1}{\xi}-D a \nabla^{2}\right)\left(R_{T} \nabla_{h}^{2} T-R_{S} \nabla_{h}^{2} S\right)
\end{aligned}
$$


S. N. Gaikwad and S. S. Kamble / JAFM, Vol. 7, No. 3, pp. 459-471, 2014.

$$
\begin{aligned}
& {\left[\frac{\partial}{\partial t}-\left(\eta \nabla_{h}^{2}+\frac{\partial^{2}}{\partial z^{2}}\right)+\boldsymbol{q} \cdot \nabla\right] T-w=0} \\
& {\left[\lambda \frac{\partial}{\partial t}-\frac{1}{L e} \nabla^{2}+\boldsymbol{q} \cdot \nabla\right] S-w=S r \frac{R_{T}}{R_{S}} \nabla^{2} T}
\end{aligned}
$$

Where $\operatorname{Pr}_{D}=\gamma \varepsilon v d^{2} / K_{z} D_{1 z}$, the Darcy-Prandtl number, $D a=\mu_{e} K_{Z} / \mu_{f} d^{2}$, the modified Darcy number, $T a=\left(2 \Omega K_{Z} / \varepsilon v\right)^{2}$, the Taylor number, $S r=D_{11} \beta_{S} / D_{1 z} \beta_{T}$, the Soret parameter $R_{T}=\beta_{T} g \Delta T d K_{z} / v D_{1 z}$, the thermal Rayleigh number, $R_{S}=\beta_{S} g \Delta S d K_{z} / v D_{1 z}$, the solute Rayleigh number, $L e=D_{1 z} / D_{2}$, the Lewis number, $\xi=K_{x} / K_{z}$, the mechanical anisotropy parameter, $\eta=D_{1 x} / D_{1 z}$, the thermal anisotropy parameter, $\lambda=\varepsilon / \gamma$, normalized porosity. The boundary conditions in only the $z$-direction are required to solving Eqs. (10) to (12) and are given by

$$
w=\frac{\partial^{2} w}{\partial z^{2}}=T=S=0 \quad \text { at } z=0
$$

\section{LINEAR STABILITY ANALYSIS}

In this section we predict the thresholds of both, stationary and oscillatory convections using linear theory. The Eigen value problem defined by Eqs. (10) to (12) subject to the boundary conditions (13) is solved using the time-dependent periodic disturbances in a horizontal plane. Assuming that the amplitudes of the perturbations are very small, we write

$$
\left(\begin{array}{l}
w \\
T \\
S
\end{array}\right)=\left(\begin{array}{l}
W(z) \\
\Theta(z) \\
\Phi(z)
\end{array}\right) \exp [i(l x+m y)+\sigma t]
$$

where $l, m$ are horizontal wavenumbers and $\sigma$ is the growth rate. Infinitesimal perturbations of the rest state may either damp or grow depending on the value of the parameter $\sigma$. Substituting Eq. (14) into the linearized version of Eqs. (10) to (12) we obtain

$$
\begin{aligned}
& w\left[\begin{array}{l}
T a D^{2}+\left(\begin{array}{l}
\frac{\sigma}{P r_{D}}\left(D^{2}-a^{2}\right)- \\
\left(D^{2}-a^{2}\right)^{2} D a+\frac{1}{\xi} D^{2}-a^{2}
\end{array}\right) \\
\left(\frac{\sigma}{P r_{D}}+\frac{1}{\xi}-\left(D^{2}-a^{2}\right) D a\right)
\end{array}\right] \\
& =\left(\begin{array}{l}
\left(\frac{\sigma}{P r_{D}}+\frac{1}{\xi}-\left(D^{2}-a^{2}\right) D a\right) \\
\left(R_{S} a^{2} \Phi-R_{T} a^{2} \Theta\right)
\end{array}\right) \\
& {\left[\sigma-\left(D^{2}-a^{2} \eta\right)\right] \Theta-W=0}
\end{aligned}
$$

$$
\left[\sigma \lambda-\frac{1}{L e}\left(D^{2}-a^{2}\right)\right] \Phi-W=B\left(D^{2}-a^{2}\right) \Theta
$$

Where, $B=S r \frac{R_{T}}{R_{S}}, \quad D=d / d z \quad$ and

$$
a^{2}=l^{2}+m^{2} \text {. }
$$

We assume the solutions of Eqs. (15) to (17) satisfying the boundary conditions (13) in the form $\left(\begin{array}{l}W(z) \\ \Theta(z) \\ \Phi(z)\end{array}\right)=\left(\begin{array}{l}W_{0} \\ \Theta_{0} \\ \Phi_{0}\end{array}\right) \operatorname{Sin} n \pi z,(n=1,2,3 \ldots \ldots)$

The most unstable mode corresponds to $n=1$ (fundamental mode). Therefore substituting Eq. (18) with $n=1$ into Eqs. (15) to (17), we obtain a matrix equation

$$
\left(\begin{array}{ccc}
A_{11} & -a^{2} R_{T} & a^{2} R_{S} \\
-1 & \sigma+\delta_{2}^{2} & 0 \\
-1 & B \delta^{2} & \sigma \lambda+\delta^{2} L e^{-1}
\end{array}\right)\left(\begin{array}{l}
W_{0} \\
\Theta_{0} \\
\Phi_{0}
\end{array}\right)=\left(\begin{array}{l}
0 \\
0 \\
0
\end{array}\right)(19)
$$

For brevity $A_{11}, \delta^{2}, \delta_{1}^{2}$ and $\delta_{2}^{2}$ are not mentioned here. The condition of nontrivial solution of above system of homogeneous linear Eqs (19) yields the expression for thermal Rayleigh number in the form

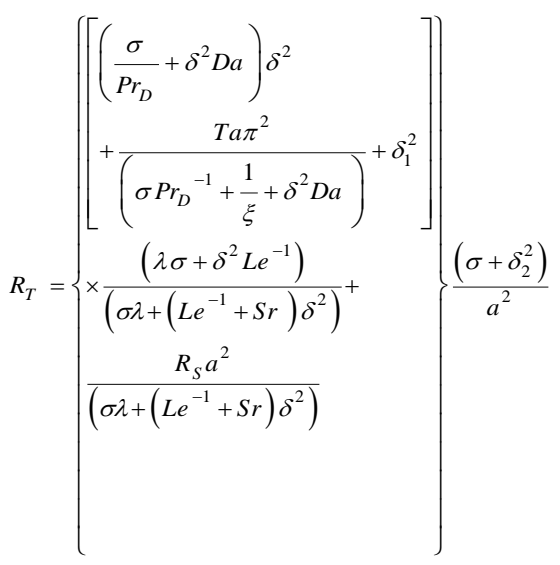

\subsection{Stationary State}

If $\sigma$ is real, then marginal stability occurs when $\sigma=0$ (i.e. $\omega_{r}=\omega_{i}=0$ ). Then the Rayleigh number $R_{T}^{S t}$ for the onset of stationary convection, in the form 


$$
\begin{aligned}
& R_{T}^{S t}=\frac{\left(a^{2} \eta+\pi^{2}\right)}{a^{2}(1+S r L e)}\left[\begin{array}{l}
\left(a^{2}+\pi^{2} \xi^{-1}\right)+ \\
\left(a^{2}+\pi^{2}\right)^{2} D a \\
+\frac{T a \pi^{2}}{\left(\xi^{-1}+\left(a^{2}+\pi^{2}\right) D a\right)}
\end{array}\right] \\
& \times \frac{\left(a^{2} \eta+\pi^{2}\right)}{(1+\text { SrLe })\left(a^{2}+\pi^{2}\right)} R_{S} L e .
\end{aligned}
$$

In the absence of Soret effect i.e., $S r=0$ Eq. (21) gives

$$
\begin{aligned}
& R_{T}^{S t}=\frac{\left(a^{2} \eta+\pi^{2}\right)}{a^{2}}\left[\begin{array}{l}
\left(a^{2}+\frac{\pi^{2}}{\xi}\right)+\left(a^{2}+\pi^{2}\right)^{2} D a \\
+\frac{T a \pi^{2}}{\frac{1}{\xi}+\left(a^{2}+\pi^{2}\right) D a}
\end{array}\right] \\
& \frac{\left(a^{2} \eta+\pi^{2}\right)}{\left(a^{2}+\pi^{2}\right)} R_{S} L e .
\end{aligned}
$$

The minimum value of the Rayleigh number $R_{T}^{S t}$ occurs at the critical wavenumber $a=a_{c}^{S t}$. In the limit as $D a \rightarrow 0$ i.e., for a densely packed porous medium Eq. (22) reduces to

$$
\begin{aligned}
& R_{T}^{S t}=\frac{\left(a^{2} \eta+\pi^{2}\right)\left[\left(a^{2}+\frac{\pi^{2}}{\xi}\right)+\pi^{2} T a \xi\right]}{a^{2}} \\
& +\left(\frac{a^{2} \eta+\pi^{2}}{a^{2}+\pi^{2}}\right) R_{S} L e .
\end{aligned}
$$

This is exactly the one given by Malashatty and Heera (2008). When $D a \rightarrow 0$ and $T a=0$, i.e. for a densely packed porous medium in the absence of rotation, Eq. (23) reduces to

$$
\begin{aligned}
& R_{T}^{S t}=\frac{1}{a^{2}}\left(a^{2}+\frac{\pi^{2}}{\xi}\right)\left(a^{2} \eta+\pi^{2}\right) \\
& +\left(\frac{a^{2} \eta+\pi^{2}}{a^{2}+\pi^{2}}\right) R_{S} L e
\end{aligned}
$$

given by Malashatty and Swamy (2009). Further, for an isotropic porous media, that is when $\xi=\eta=1$, Eq. (22) gives

$R_{T}^{S t}=\frac{\pi^{4}\left(1+\alpha^{2}\right)^{3} M}{\alpha^{2}}+\frac{T a}{M \alpha^{2}}+\frac{R_{S}}{\tau}$,

Where

$$
M=\frac{1}{\pi^{2}}\left(D a \pi^{2}+\left(1+\frac{a^{2}}{\pi^{2}}\right)^{-1}\right), \alpha^{2}=\frac{a^{2}}{\pi^{2}}, \tau=\frac{1}{L e},
$$

which is the one obtained by Rudraiah et al. (1986).

\subsection{Oscilatory State}

We now set $\sigma=i \omega_{i}$ in Eq. (20) and clear the complex quantities from the denominator, to obtain

$R_{T}=\Delta_{1}+i \omega_{i} \Delta_{2}$

Since $R_{T}$ is a physical quantity, it must be real. Hence, from Eq. (26) it follows that either $\omega_{i}=0$ (steady onset) or $\Delta_{2}=0 \quad\left(\omega_{i} \neq 0\right.$, oscillatory onset).

For oscillatory onset $\Delta_{2}=0 \quad\left(\omega_{i} \neq 0\right)$ and this gives a dispersion relation of the form (on dropping the subscript $i$ )

$a_{0}\left(\omega^{2}\right)^{2}+a_{1}\left(\omega^{2}\right)+a_{2}=0$

for simplicity the coefficients $a_{0}, a_{1}$ and $a_{2}$ are not mentioned here. Now Eq. (26) with $\Delta_{2}=0$, gives

$$
\begin{aligned}
& R_{T}^{o s c}=\frac{\left(\operatorname{Ta\xi }\left(\begin{array}{c}
\omega^{2}\left(\begin{array}{c}
P_{r_{D} r_{1} \delta^{4} \xi+\lambda L e^{2} \times}\left(\omega^{2} \xi \lambda-P r_{D}^{2} r_{2} S r \delta^{2}\right)
\end{array}\right)+ \\
\delta_{2}^{2}\left(\begin{array}{l}
\omega^{2} \delta^{2} L e P r_{D} r_{1} \lambda \xi \\
-\omega^{2} L e \delta^{2} \lambda \xi+P r_{D}^{2} \times \\
\left(\delta^{4} r_{1}+\omega^{2} L e^{2} \lambda^{2}\right) r_{2}
\end{array}\right)
\end{array}\right) \pi^{2}\right)}{\left(a^{2}\left(\delta^{4} r_{1}^{2}+\omega^{2} L e^{2} \lambda^{2}\right)\left(P_{D}^{2} r_{2}^{2}+\omega^{2} \xi^{2}\right)\right)}+ \\
& \left(-\omega \delta^{2}\left(\begin{array}{l}
\omega^{2} L e^{2} \lambda^{2}+\delta^{4}\left(r_{1}\left(1+\operatorname{DaLePr}_{D} \lambda\right)\right) \\
+L e^{2} \operatorname{Pr}_{D} S r \lambda \delta_{1}^{2}
\end{array}\right)+\right. \\
& \left(\delta^{4}\left(\begin{array}{l}
-\omega^{2} L e^{2} S r \lambda+D a P r_{D} \times \\
\left(\delta^{4} r_{1}+\omega^{2} L e^{2} \lambda^{2}\right)
\end{array}\right)+\right) \delta_{2}^{2} \\
& \frac{\left(\operatorname{Pr}_{D}\left(\delta^{4} r_{1}+\omega^{2} L e^{2} \lambda^{2}\right) \delta_{1}^{2}\right)}{\left(a^{2}\left(\delta^{4} r_{1}^{2}+\omega^{2} L e^{2} \lambda^{2}\right) P r_{D}\right)} \\
& +\frac{L e R_{S}\left(\delta_{2}^{2} \delta^{2} r_{1}+\omega^{2} L e \lambda\right)}{\left(\delta^{4} r_{1}^{2}+\omega^{2} L e^{2} \lambda^{2}\right)}
\end{aligned}
$$

\section{RESULT AND DISCUSSION}

The Soret effect on the onset of double-diffusive rotating anisotropic convection in a horizontal sparsely packed porous layer is investigated theoretically using the linear stability theory. The variation of stationary and oscillatory curves in the $R_{T}-a$ plane for various parameter values are as shown in Figs.(1a to 4d). We fixed the values for the parameters except the varying parameter.

Figs (1a to 1d) and (3a to $3 \mathrm{~d}$ ) shows that the stationary and oscillatory curves respectively for different values of $\xi, \eta, T a$ and $D a$ for fixed values of other parameters. We observed from these figures that the convection sets in as oscillatory mode prior to the stationary mode. 


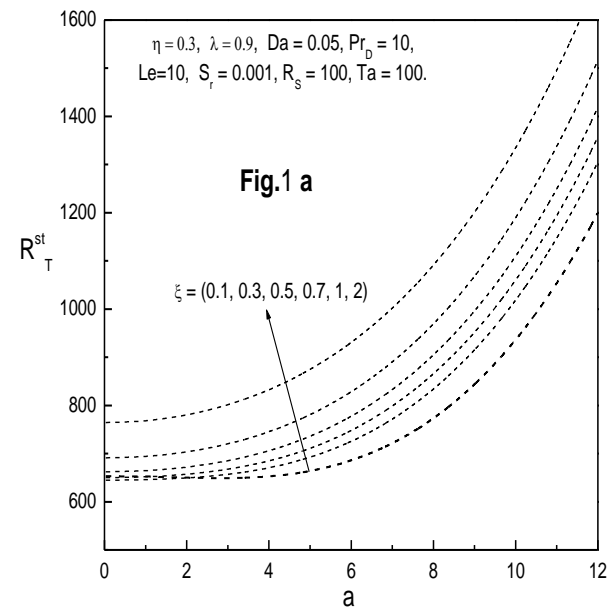

Fig. 1a. Variation of stationary curves for different values of $\xi$

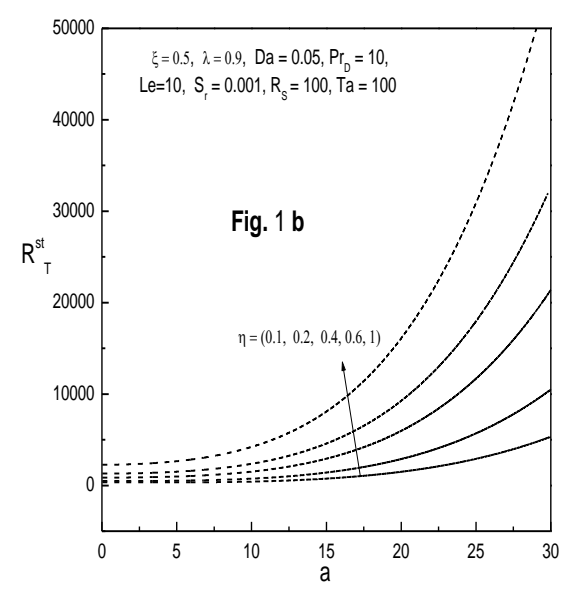

Fig. 1b. Variation of stationary curves for different values of $\eta$

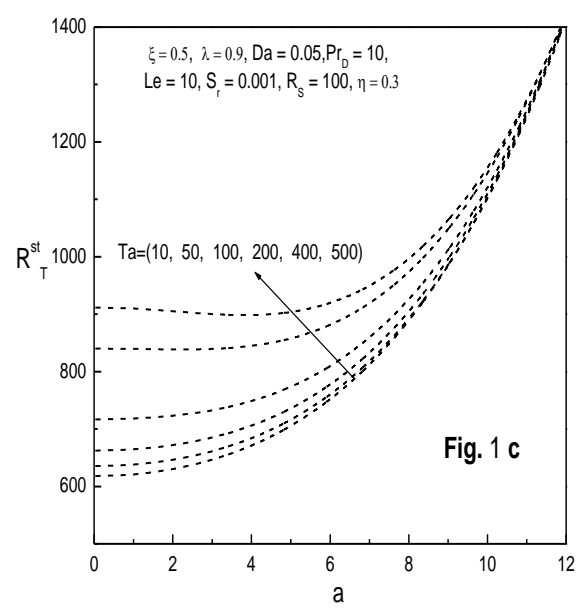

Fig. 1c. Variation of stationary curves for different values of $T a$

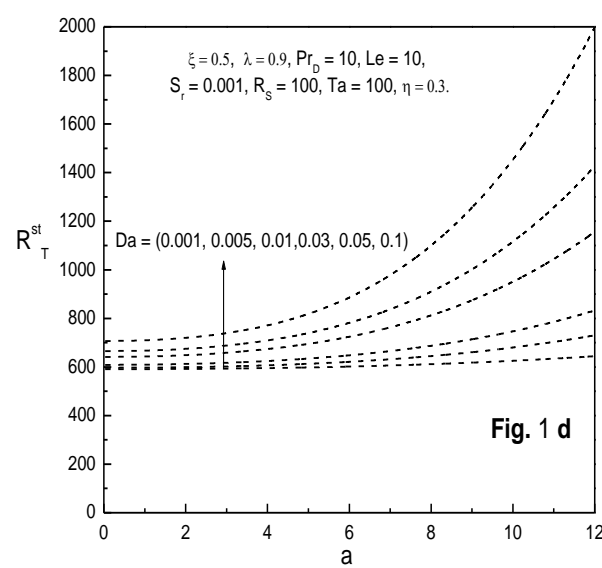

Fig. 1d. Variation of stationary curves for different values of $D a$

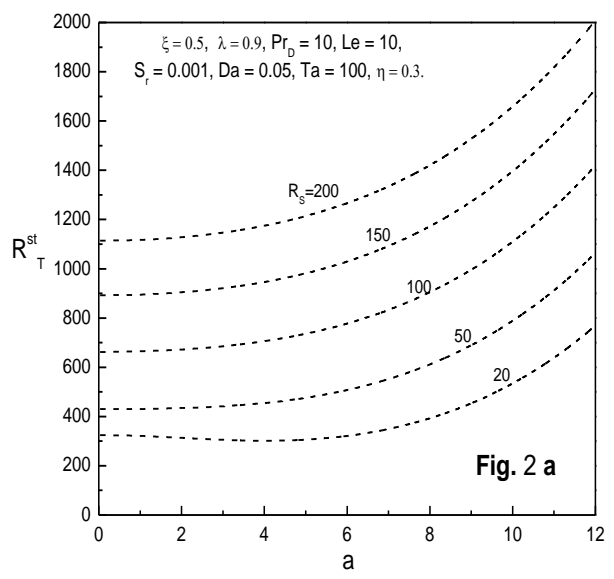

Fig.2a. Variation of stationary curves for different values of $R_{S}$

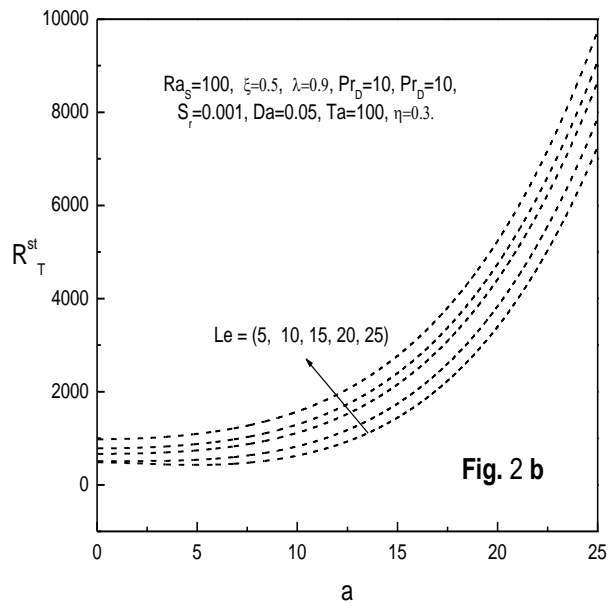

Fig. 2b. Variation of stationary curves for different values of $L e$ 


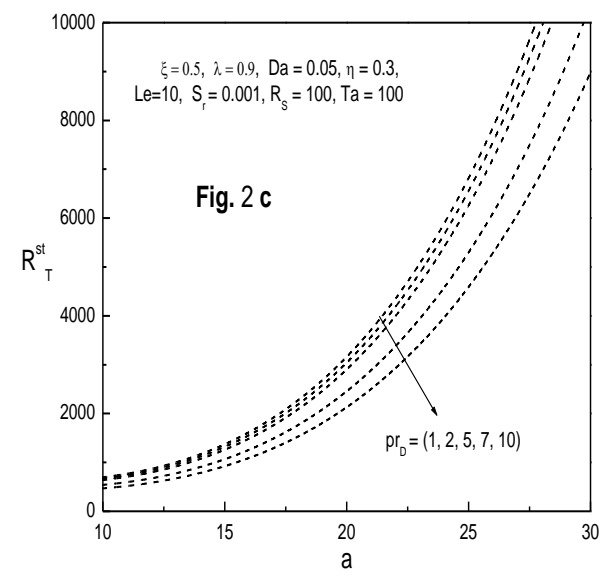

Fig. 2c. Variation of stationary curves for different values of $\operatorname{Pr}_{D}$

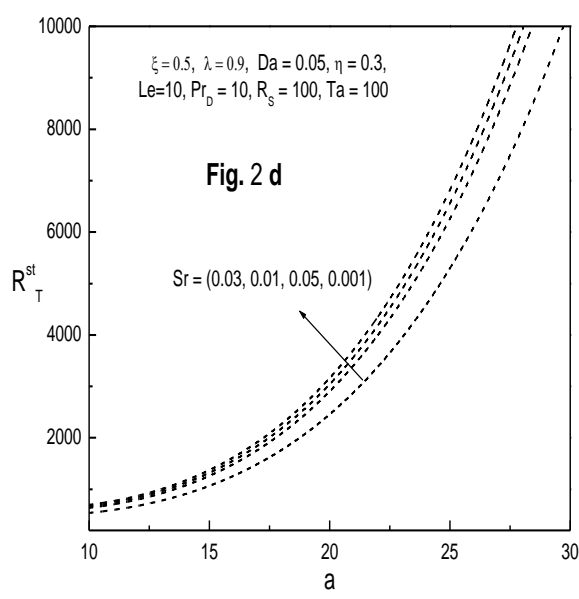

Fig.2d. Variation of stationary curves for different values of $\mathrm{Sr}$

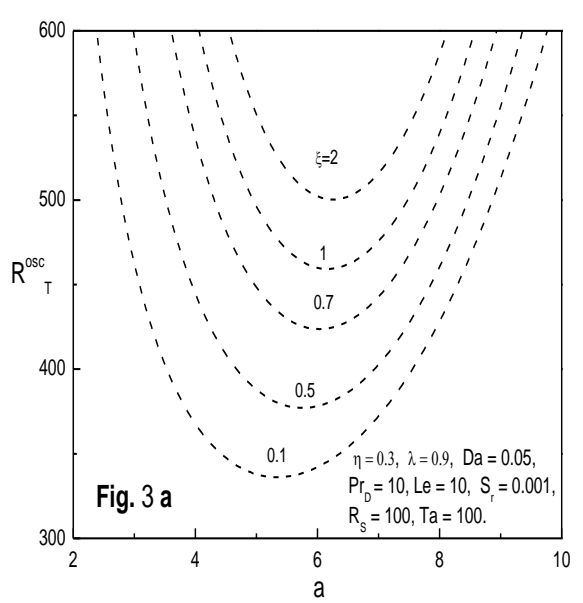

ig. 3a. Variation of oscillatory curves for different values of $\xi$

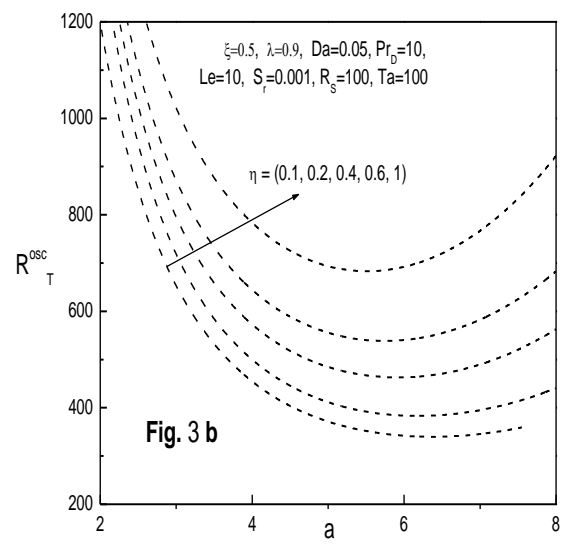

Fig. 3b. Variation of oscillatory curves for different values of $\eta$

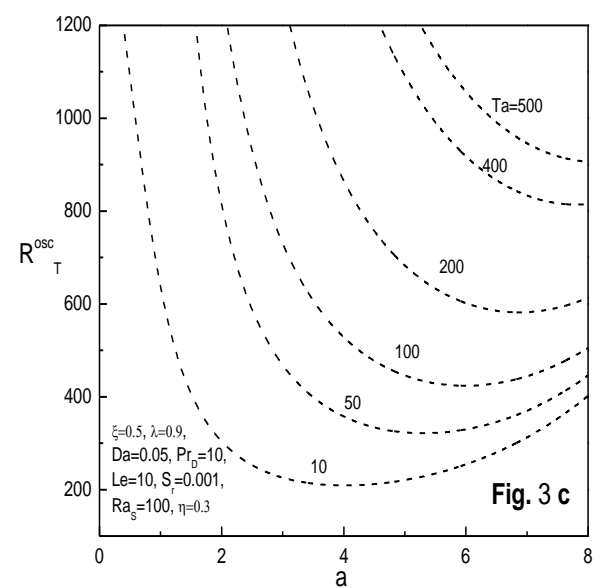

Fig. 3c. Variation of oscillatory curves for different values of $T a$

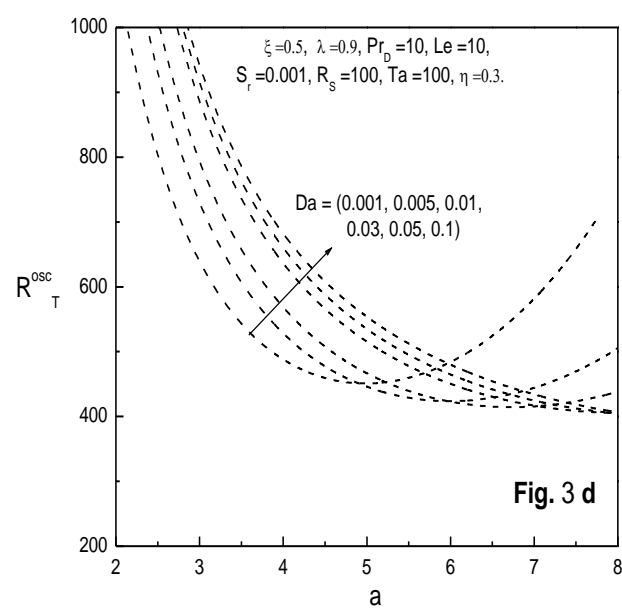

Fig. 3d. Variation of oscillatory curves for different values of $D a$

From Figs. 1a and 3a, it can be observed that the critical value of the Rayleigh number increases with 
the increasing $\xi$ for both oscillatory and stationary convection. Thus $\xi$ has stabilizing effect on stationary and oscillatory convection. Figs. $1 \mathrm{~b}$ and $3 b$ indicates the effect of thermal anisotropy parameter $\eta$ on both the stationary and oscillatory convection for the fixed values of other parameters. It is observed that critical value of the Rayleigh number for stationary and oscillatory mode increases with increasing $\eta$, indicating that the effect of $\eta$ is to inhibit the onset of stationary and oscillatory convection. Figs. $1 \mathrm{c}$ and $3 \mathrm{c}$ depicts the effect of Taylor number $\mathrm{Ta}$ on the both the stationary and oscillatory curves. We find that the effect of increasing $T a$ is to increase the critical value of the Rayleigh number for stationary and oscillatory modes and the corresponding wavenumber. Thus the Taylor number $\mathrm{Ta}$ has a stabilizing effect on the double diffusive convection in a horizontal sparsely packed anisotropic porous medium. Figs. 1d and 3d present the effect of Darcy number $D a$ on both the curves. We find that critical value of the stationary Rayleigh number increases with $D a$, indicating that the effect of Darcy number $D a$ is to inhibit the onset of stationary convection. Whereas for the oscillatory convection, the critical value of oscillatory Rayleigh number decreases with increasing $D a$, and with further increase in the value of $D a$, the critical Rayleigh number increases. Thus $D a$ has dual effect on oscillatory convection.

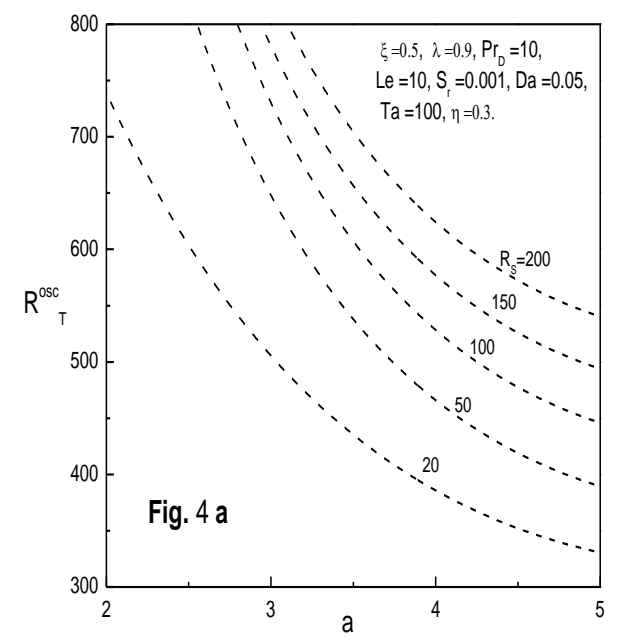

Fig. 4a. Variation of oscillatory curves for different values of $R_{S}$

Figs. (2a to $2 \mathrm{~d}$ ) and (4a to $4 \mathrm{~d}$ ) depict the effect of $R_{S}, L e, \operatorname{Pr}_{D}$ and $S r$ on stationary and oscillatory modes respectively. We find from Figs. 2a and $4 \mathrm{a}$ that the effect of increasing $R_{S}$ is to increase the critical value of the Rayleigh number for stationary and oscillatory modes and the corresponding wavenumber. Thus the solute Rayleigh number $R_{S}$ has a stabilizing effect on the double diffusive convection in a horizontal sparsely packed anisotropic porous medium.

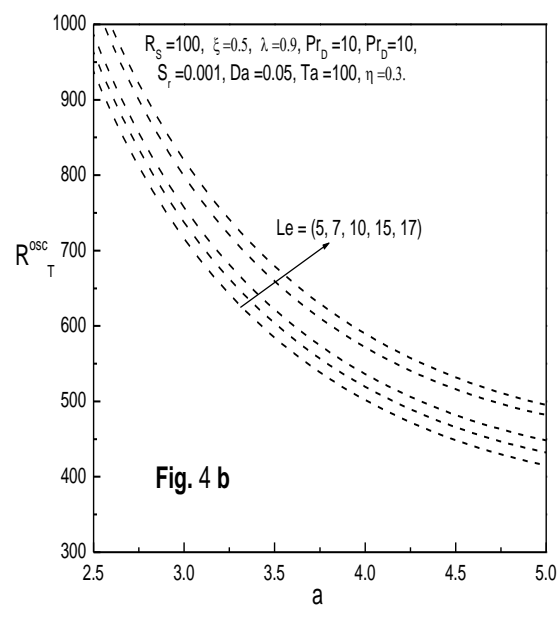

Fig. 4b. Variation of oscillatory curves for different values of $L e$

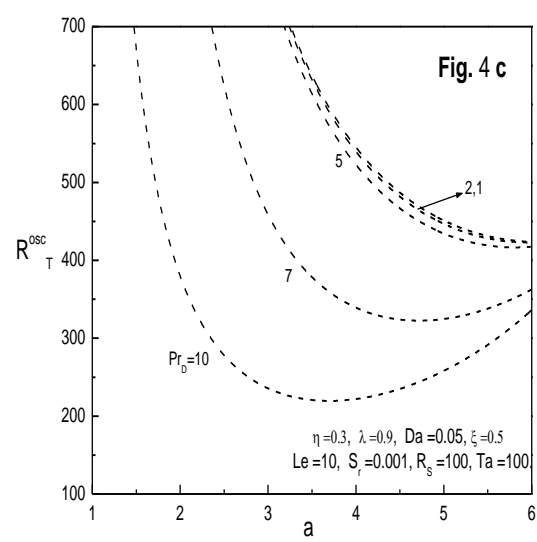

c. Variation of oscill values of $\operatorname{Pr}_{D}$

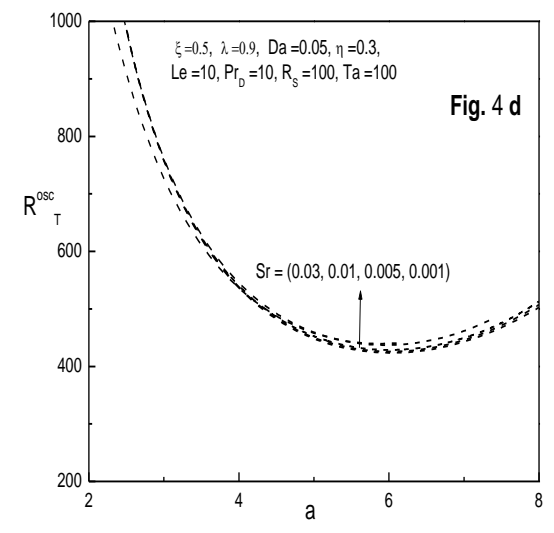

Fig. 4d. Variation of oscillatory curves for different values of $\mathrm{Sr}$ 
In Figs. $2 \mathrm{~b}$ and $4 \mathrm{~b}$ the marginal and oscillatory curves for different values of Lewis number $L e$ are drawn. It is observed that with the increase of $L e$ the critical values of Rayleigh number and the corresponding wavenumber for oscillatory mode decreases while those for stationary mode increases. Therefore, the effect of $L e$ is to advance the onset of oscillatory convection while its effect is to inhibit the stationary convection. The marginal and oscillatory curves for different values of DarcyPrandtl number $\operatorname{Pr}_{D}$ are presented in Figs.2c and $4 \mathrm{c}$, from these figures it is evident that for small and moderate values of $\operatorname{Pr}_{D}$ the critical value of oscillatory Rayleigh number decreases with the increase of $\operatorname{Pr}_{D}$, however this trend is reversed for large values of $\operatorname{Pr}_{D}$. Figures. $2 \mathrm{~d}$ and $4 \mathrm{~d}$ indicates the effect of Soret parameter $\mathrm{Sr}$ on the both the stationary and oscillatory modes. It is observed that as $\mathrm{Sr}$ increases positively, the critical values of Rayleigh number and the corresponding wavenumber for oscillatory mode increase while as $\mathrm{Sr}$ increases negatively, those decrease. Whereas the effect is reversed for the stationary mode. Thus Soret parameter has stabilizing effect on oscillatory convection and destabilizing effect on stationary convection.

The detailed behavior of stationary and oscillatory critical Rayleigh number with respect to the Taylor number is analyzed in the $R_{T c}-T a$ plane through Figs. (5a to $5 \mathrm{f}$ ) and (6a to $6 \mathrm{f}$ ) respectively. We observe from these figures that the critical Rayleigh number increases with the increase of $T a$, indicating that the effect of rotation is to inhibit the onset of thermal convection and it is in agreement with the corresponding problem of isotropic and pure fluid layer.

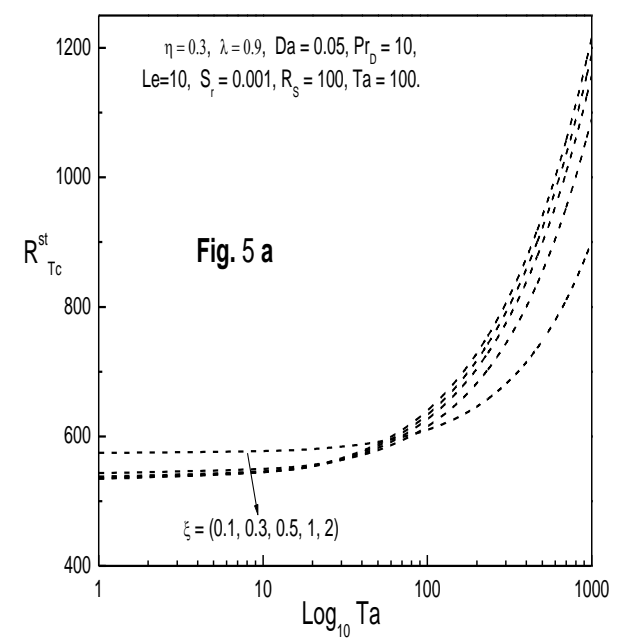

Fig. 5a. Variation of stationary critical curves for different values of $\xi$

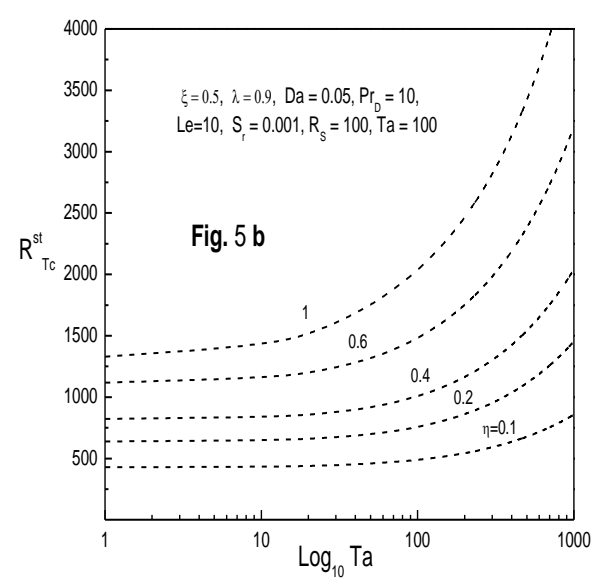

Fig. 5b. Variation of stationary critical curves for different values of $\eta$

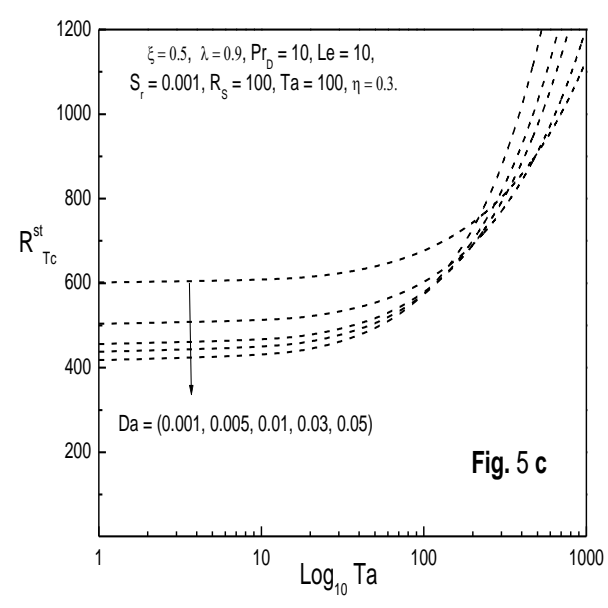

Fig. 5c. Variation of stationary critical curves for different values of $D a$

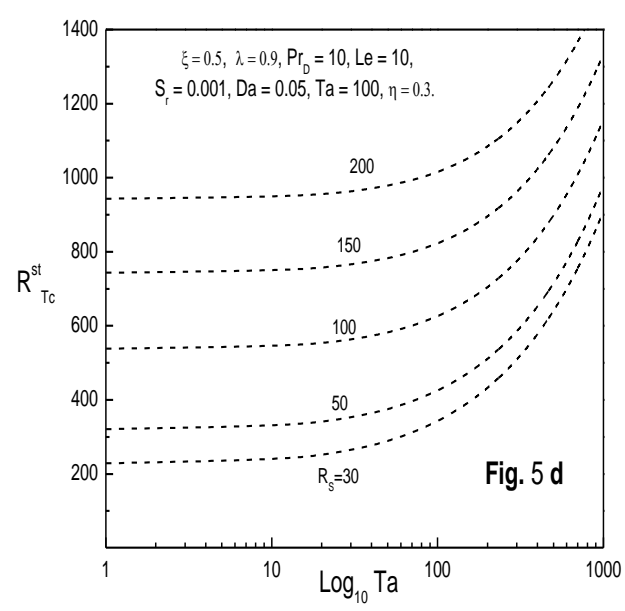

Fig. 5d. Variation of stationary critical curves for different values of $R_{S}$ 


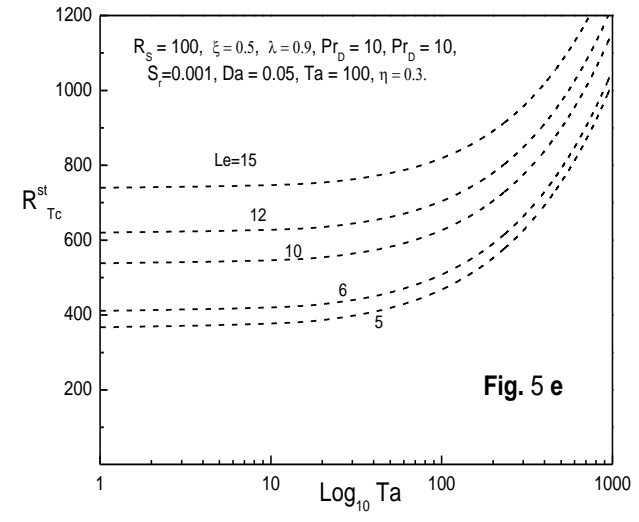

Fig. 5e. Variation of stationary critical curves for different values of $L e$

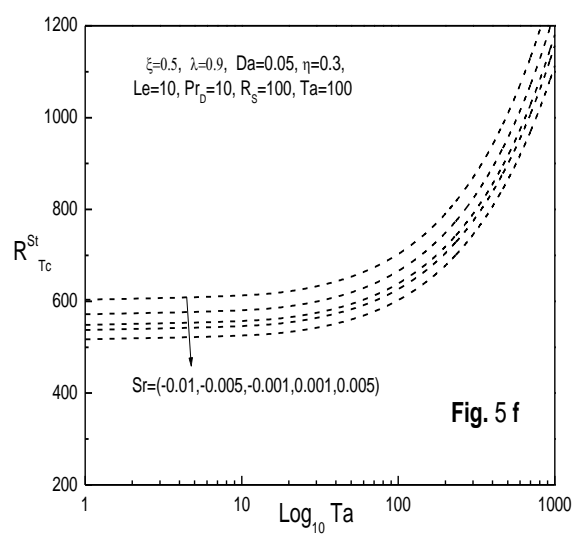

Fig. 5f. Variation of stationary critical curves for different values of $\mathrm{Sr}$

In Figs. 5a and 6a, we display the variation of stationary and oscillatory critical Rayleigh number $R_{T c}$ with Taylor number $T a$ for different values of mechanical anisotropy parameter $\xi$ for the fixed values of other parameters. It is important to note that $R_{T c}$ decreases with the increase of $\xi$ for small values of $T a$, and for large value of $T a$, the critical Rayleigh number increases. Thus the mechanical anisotropy parameter $\xi$ has dual effect on oscillatory and stationary convection. Figures. $5 \mathrm{~b}$ and $6 \mathrm{~b}$ indicates the variation of $R_{T c}$ with $T a$ for different values of thermal anisotropy parameter $\eta$. It is observed that the critical Rayleigh number $R_{T c}$ increases with the increase of $\eta$ indicating that the effect of thermal anisotropy parameter is to inhibit the onset of stationary and oscillatory convection.

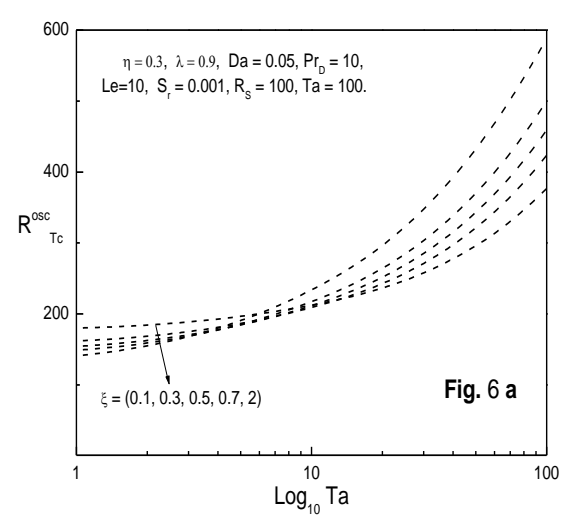

Fig. 6a. Variation of oscillatory critical curves for different values of $\xi$

Figures. $5 \mathrm{c}$ and $6 \mathrm{c}$ presents the variation of $R_{T c}$ with $T a$ for different values of Darcy number $D a$. We find that the critical Rayleigh number $R_{T C}$ increases with the increase of $D a$ for small values of $T a$, and for large value of $T a$, the critical Rayleigh number decreases. Thus $D a$ has dual effect on oscillatory and stationary convection. The variation of $R_{T c}$ with $T a$ for different values of solute Rayleigh number $R_{S}$ on the onset criteria is shown in Figs. 5d and 6d. We observe from these figures that $R_{T c}$ increases with the increasing $R_{S}$. Thus the effect of $R_{S}$ is to inhibit the onset of convection for both stationary and oscillatory modes. In Figs. 5e and 6e, the variation of $R_{T c}$ with $T a$ for different values of Lewis number $L e$ is shown for the fixed values of other parameters. It is observed that $R_{T C}$, increases with the increase of $L e$, for the stationary convection while decreases for the oscillatory convection, indicating that $L e$ has stabilizing effect on stationary convection while destabilizing effect on oscillatory convection.

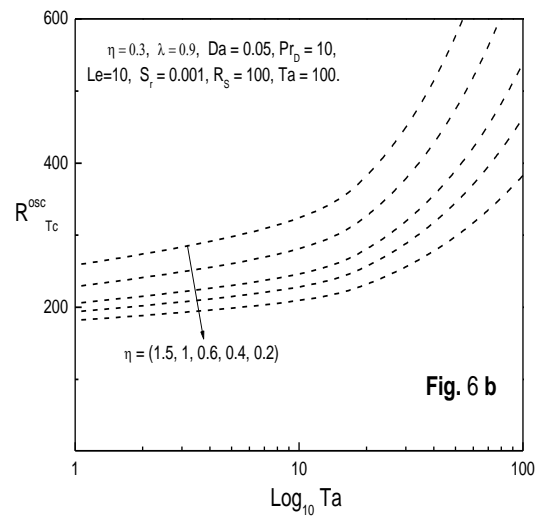

Fig. 6b. Variation of oscillatory critical curves for different values of $\eta$ 


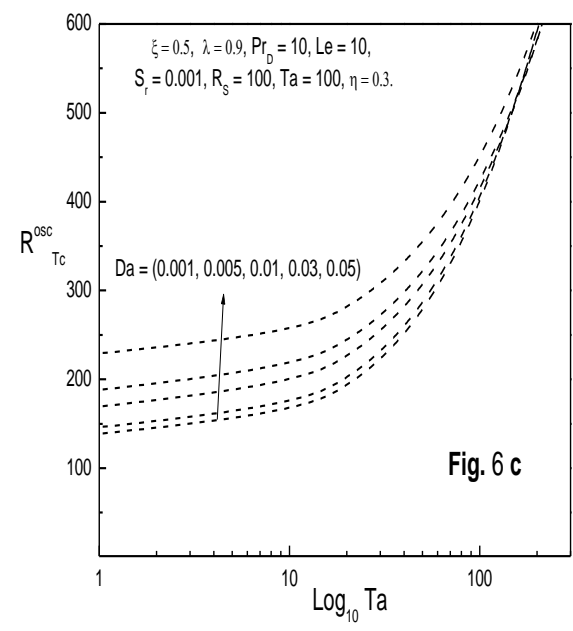

Fig. 6c. Variation of oscillatory critical curves for different values of $D a$

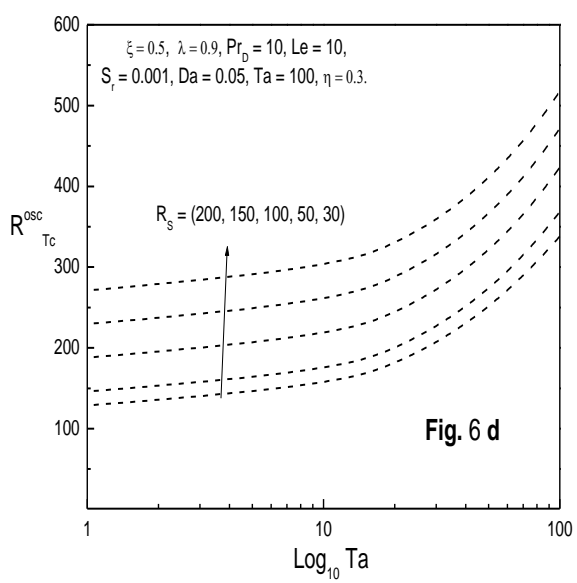

Fig. 6d. Variation of oscillatory critical curves for different values of $R_{S}$

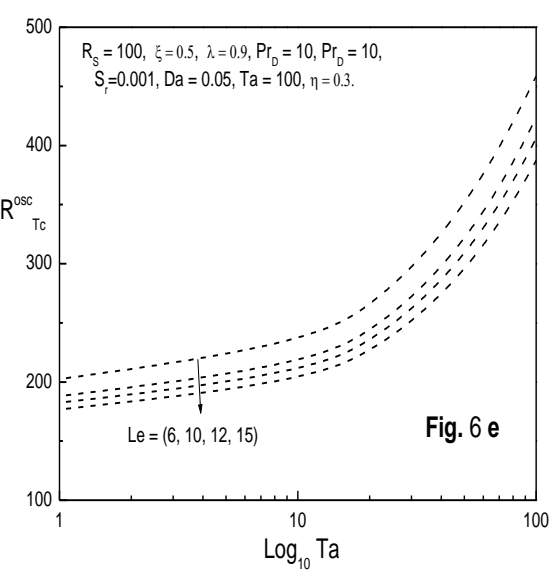

Fig. 6e. Variation of oscillatory critical curves for different values of $L e$

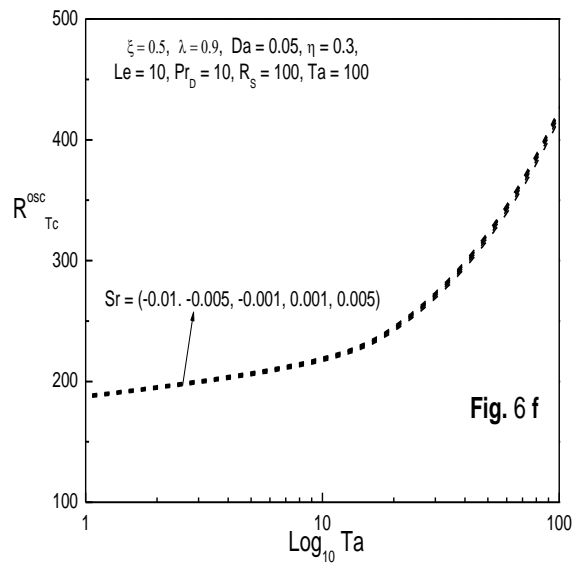

Fig. 6f. Variation of oscillatory critical curves for different values of $S r$

The variation of $R_{T c}$ with $T a$ for different values of Soret parameter is presented in the Figs. $5 \mathrm{f}$ and 6f. We find that as $S r$ increases positively, the critical values of Rayleigh number and the corresponding wavenumber decrease for stationary mode while as $\mathrm{Sr}$ increases negatively, those increase. And the effect of $S r$ is reversed for oscillatory mode. Thus positive $\mathrm{Sr}$ has destabilizing effect for stationary mode and stabilizing effect for oscillatory mode.

\section{CONCLUSIONS}

The effect of rotation on the onset of doublediffusive convection in a horizontal sparsely packed anisotropic porous layer, in the presence of Soret effect is investigated theoretically using the linear stability theory. The usual normal mode technique is used to solve the linear problem. The applications of the present study has recently increased in view of its potential occurrence in nature and wide range of applications such as highquality crystal production, liquid gas storage, migration of moisture in fibrous insulation, transport of contaminants in saturated soil, solidification of molten alloys, and geothermally heated lakes and magmas, underground disposal of nuclear wastes, liquid re-injection, electro-chemical and drying processes

Finally, from the results we conclude that the mechanical anisotropy parameter $\xi$ has stabilizing effect on stationary and oscillatory. However the convection sets in as oscillatory mode prior to the stationary mode. The effect of thermal anisotropy parameter $\eta$ is to inhibit the onset of stationary and oscillatory convection. The Taylor number $T a$ has a stabilizing effect on the double diffusive convection in sparsely packed anisotropic porous medium. The effect of Darcy number $D a$ is to inhibit the on the onset of stationary convection while it has dual effect on oscillatory convection. The effect of solute Rayleigh number is to delay 
both stationary and oscillatory convection. And the effect of Lewis number is to delay the onset of stationary convection while it advances the oscillatory convection. The Darcy Prandtl $\operatorname{Pr}_{D}$ has a dual effect on the oscillatory mode. The Soret parameter has stabilizing effect on oscillatory convection and destabilizing effect on stationary convection.

\section{REFERENCES}

Bahloul, A., Boutana, N. and Vasseur, P. (2003). Double diffusive and Soret-induced convection in a shallow horizontal porous layer, J. Fluid Mech. Vol. 491, pp. 325-352.

Bennacer, R., Mohamad, A.A. and Ganaoui, M. El. (2005). Analytical and numerical investigation of double diffusion in thermally anisotropy multilayer porous medium, Heat Mass Transfer.. 41, 298 -305.

Brand H. R. and Steinberg V. (1983). Convective instabilites in binary mixtures in a porous medium. Physica, A 119, 327-357.

Castinel, G. and Combarnous, M. (1974). Critere d'apparition de la convection naturelle dans une couche poreuse anisotrope horizontal, C.R. Acad. Sci B, 278, 701-704

Chakrabarti,A., and Gupta, A.S. (1981). Nonlinear thermohaline convection in a rotating porous medium, Mech.Res.Commun. 8, 9-15.

Chen, C.F. and Chen, F. (1993). Double-diffusive fingering convection in a porous medium, Int J. Heat Mass Transfer, 36, 793-807.

Epherre, J.F. (1977). Criterion for the appearance of natural convection in an anisotropic porous layer, Int. Chem. Engng. 17, 615-616

Gaikwad S.N. and Kamble S.S.(2012). Soret effect on linear DDC in a horizontal sparsely packed porous layer, IJPMS , vol. 2(2) , 97104Govender, S. (2007). Coriolis effect on the stability of centrifugally driven convection in a rotating anisotropic porous layer subjected to gravity, Transport in Porous Media, 67, 219227.

Hill, A.A. (2005). Double-diffusive convection in a porous medium with a concentration based internal heat source, Proc. R. Soc. A. 461, 561-574.

Ingham, D.B. and Pop. I. (2005). Transport Phenomena in Porous Media, Elsevier, Oxford, Knobloch. E (1986). Oscillatory convection in binary mixtures, Phys. Rev. A, 34, 538-1549

Malashetty, M.S. (1993). Anisotropic thermo convective effects on the onset of double diffusive convection in a porous medium, Int J. Heat Mass Transfer 36, 397- 2401.

Malashetty, M. S. and Mahantesh, Swamy. (2007). The effect of rotation on the onset of convection in a horizontal anisotropic porous layer, Int. J. Thermal Sci., 46, 1023-1032.

Malashetty, M.S. and Heera, R. (2008). The effect of rotation on the onset of double diffusive convection in a horizontal anisotropic porous layer, Transp. Porous Med., 74, 105-127.

Malashetty, M.S. and Swamy, M. (2010). The onset of convection in a binary fluid saturated anisotropic porous payer, Int. J. Therm. Sci., $49,861-878$.

Malashetty M.S. and Begum I. (2011). The effect of rotation on the onset of double diffusive convection in a sparsely packed anisotropic porous layer, Transp. Porous Med., 88 , 315345 .

Mamou, M. (2002). Stability analysis of double diffusive convection in porous enclosures, In Transport Phenomena in Porous Media II Ingham D.B. and Pop, I. eds) Elsevier, Oxford, 113-154,

Mansour, A., Amahmid, A., Hasnaoui, M. and Bourich, M. (2006). Multiplicity of solutions induced by thermosolutal convection in a square porous cavity heated from below and submitted to horizontal concentration gradient in the presence of Soret effect, Numerical Heat Transfer, Part A, 49, 69-94.

McKibbin, R. (1992). Convection and heat transfer in layered and anisotropic porous media, In Heat and Mass Transfer in Porous Media, 327-336 (eds. M. Quintard and M. Todorovic), Elsevier, Amsterdam

Mojtabi, A. and Charrier-Mojtabi, M.C. (2005). Double-diffusive convection in porous media, In Handbook of Porous media (K.Vafai, ed.), $2^{\text {nd }}$ ed., Taylor and Francis, New York, 269320 .

Murray, B.T. and Chen, C.F. (1989). Double diffusive convection in a porous medium, $J$. Fluid Mech. 201, 147-166.

Nield, D. A. and Bejan, A. (2006). Convection in Porous Media, $3^{\text {rd }}$ ed., Springer-Verlag, New York.

Prabhamani, R. Patil., Parvathy, C.P. and Venkatakrishnan K.S. (1989). Thermohaline instability in a rotating anisotropic porous medium, Appl. Sci. Res. 46, 73-88. 
Rudraiah, N. and Masuoka, T. (1982). Asymptotic analysis of natural convection through horizontal pours layer. Int. J. Engng. Sci. .20, 27-39.

Rudraiah, N.and Malashetty, M.S. (1986). The influence of coupled molecular diffusion on the double diffusive convection in a porous medium, ASME J. Heat Transfer 108, 872876.

Storesletten, L. (2004). Effects of anisotropy on convection in horizontal and inclined Porous layers, In Emerging Technologies and Techniques in Porous Media, 285-306. (eds. D.B.Ingham et al.), Kluwer Academic Publishers, Netherland.

Straughan, B. and Hutter, K. (1999). A priori bounds and structural stability for double diffusive convection incorporating the Soret effect, Proc. R. Soc. Lond. A 455, 767-777.
Trevisan, O.V. and Bejan, A. (1999). Combined heat and mass transfer by natural convection in a porous medium, Advances in Heat Transfer 20, 315- 352.

Tyvand, P.A. (1980). Thermohaline instability in anisotropic porous media, Water Resour. Res., $16,325-330$.

Vafai, K. (2005). Handbook of Porous Media, Taylor and Francis/CRC Press, London/Boca Raton, FL.

Vadasz, P (2008). Emerging Topics in Heat and Mass Transfer in Porous Media, Springer, New York.

Rani H.P. and Reddy G.J. (2013). Soret and Dufour Effects on Transient Double Diffusive Free Convection of Couple-stress Fluid past a Vertical Cylinder, Journal of Applied Fluid Mechanics, 6(4), 545-554. 